Scientific Visualization, 2020, volume 12, number 1, pages 70 - 82, DOI: 10.26583/sv.12.1.06

\title{
Visualization of quality of 3D tomographic images in construction of digital rock model
}

\author{
A.S. Kornilovi,A,B, I.A. Reimers, ${ }^{2, A, C}$, I.V. Safonov3,A, I.V. Yakimchuk4,A \\ A Schlumberger Moscow Research Center \\ B National Research Nuclear University MEPhI \\ C Moscow Institute of Physics and Technology (National Research University) \\ 1 ORCID: 00oo-0002-3651-0809, kranton94@mail.ru \\ 2 ORCID: 0000-0003-1086-7081, irene.ab18@gmail.com \\ 3 ORCID: 000o-0002-8270-943X, isafonov@slb.com \\ 4 ORCID: 0000-0003-2494-0542, iyakimchuk@slb.com
}

\begin{abstract}
Various types of tomography are widely employed in oil and gas industry for studying structure of rocks. Using X-ray or FIB-SEM tomography, a 3D model of a core sample is constructed for mathematical simulations of fluid flow in porous media and evaluation of physical characteristics of rock. Since images have various defects and distortions, there is a problem of selection of a fragment with the best quality from the initial $3 \mathrm{D}$ image. At the moment this operation is made manually on the basis of an expert's opinion and takes significant time. In this paper, we investigate applicability of existing non-reference quality metrics for evaluation of tomographic images and propose the approach for visualization of spatial change of $3 \mathrm{D}$ image quality. The method includes the construction of central cross-section; plotting graphs of quality and similarity measures for each slice over the cross-section; generation of combined heat map of quality of cubic fragments with various size. The proposed approach significantly accelerates and makes less subjective selection of the best region for further simulations in digital rock workflow. The choice of colour scale is considered to facilitate the analysis of graphical information for people with colour vision deficiency.
\end{abstract}

Keywords: referenceless assessment of image quality, quality visualization, X-ray tomography, microCT, FIB-SEM, digital rock, heat map, similarity index, colour vision deficiency.

\section{Introduction}

One of the important tasks in the oil and gas industry is to assess the permeability and mechanical properties of rocks. These parameters are often evaluated using mathematical simulations, for example, numerical modelling of the multiphase fluid flow in pore space. The result of calculations directly depends on the accuracy of the three-dimensional model of oilbearing rock, the so-called digital rock $[1,2]$. This model is usually built from computed X-ray [3] and FIB-SEM [4] tomography images. These two technologies are based on various physical principles and are used to study the internal material structure at different scales.

X-ray computed microtomography (MicroCT) belongs to non-destructive techniques of visualization of the internal structure of objects. The sample is irradiated by an X-ray beam from various sides. In practice, this is implemented with rotating the object. After passing through the substance, the beam intensity decreases in accordance with the spatial distribution of the absorption capacity in the sample and is registered by the detector (figure 1a). A 3D image of the object is restored from a set of obtained shadow projections (figure 1b). The spatial resolution of modern laboratory X-ray MicroCT systems can be about $1 \mu \mathrm{m}$. 
The FIB-SEM setup uses a combination of focused ion beam (FIB) and scanning electron microscope (SEM) (figure2). The ion beam removes a thin layer of substance from the sample, and then the electron microscope takes an image of the surface. Multiple repetition of these two operations produces a set of sequential images of the sample layers. This technology can resolve details with size up to 5-10 nanometers and is widely used to study the nanostructure of not only rocks, but also other objects, such as fuel cell electrodes, semiconductors, nanomaterials, alloys, and biological tissues [4].

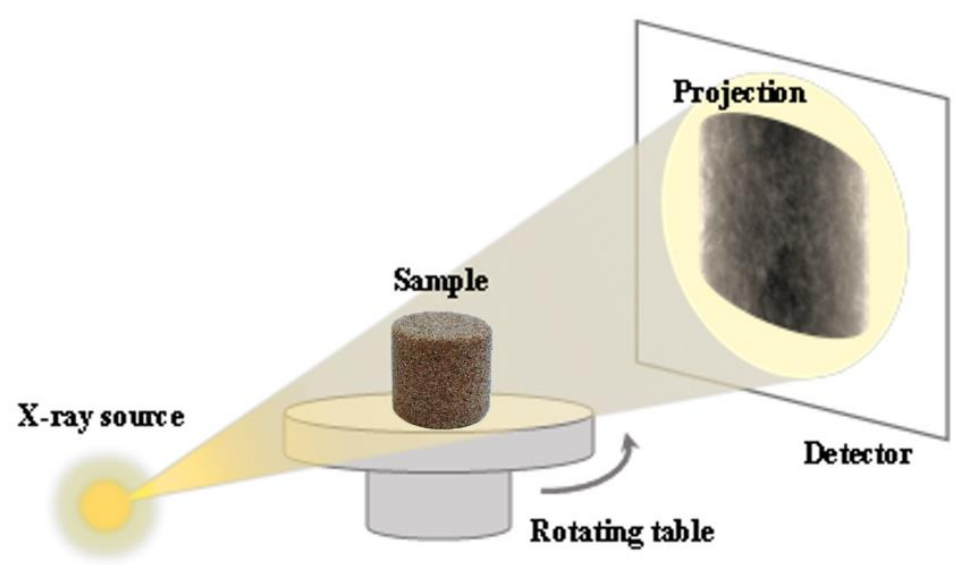

a)

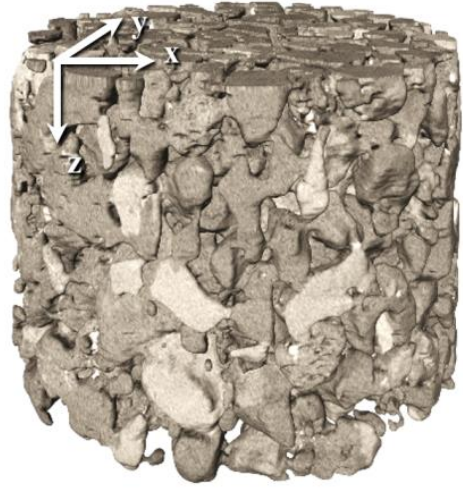

b)

Figure 1. a) X-ray microtomography scheme; b) restored three-dimensional image.

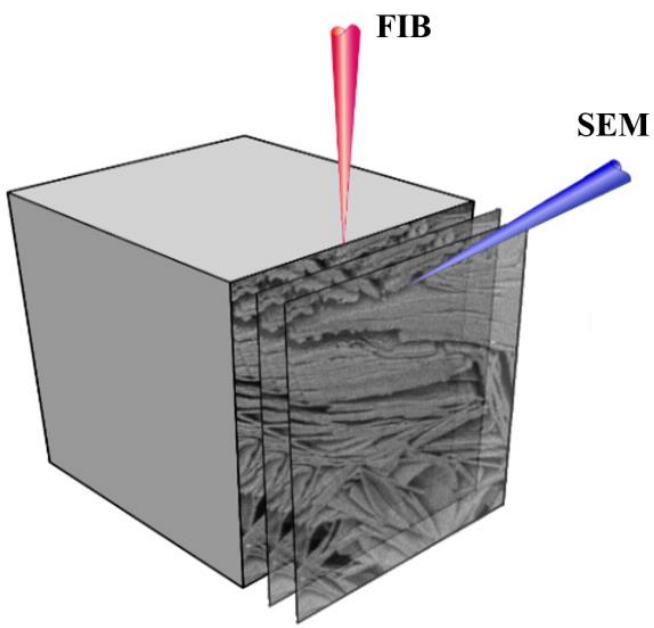

Figure 2. FIB-SEM tomography scheme.

Both X-ray and FIB-SEM images have distortions and artefacts. Defects can be typical for any raster image (for example, high noise, blur and low contrast) or specific to the particular image acquisition technology.

For example, ring-shaped artefacts may occur in microCT images (figure 3). In addition, the sample can have high-density regions that appear as bright, overexposed areas with local intensity distortions around them [3]. The artefacts in the form of high-intensity regions are also found in FIB-SEM images due to local accumulation of electric charge on the sample surface. Outwardly similar effect sometimes occurs at the pore boundaries, where the probability to emit secondary electrons is higher than inside the mineral matrix (indicated by the dashed line in figure). Another common artefact of FIB-SEM images is vertical stripes (the so-called "curtaining") arising due to deflection of the ion beam during etching the sample (figure4). 


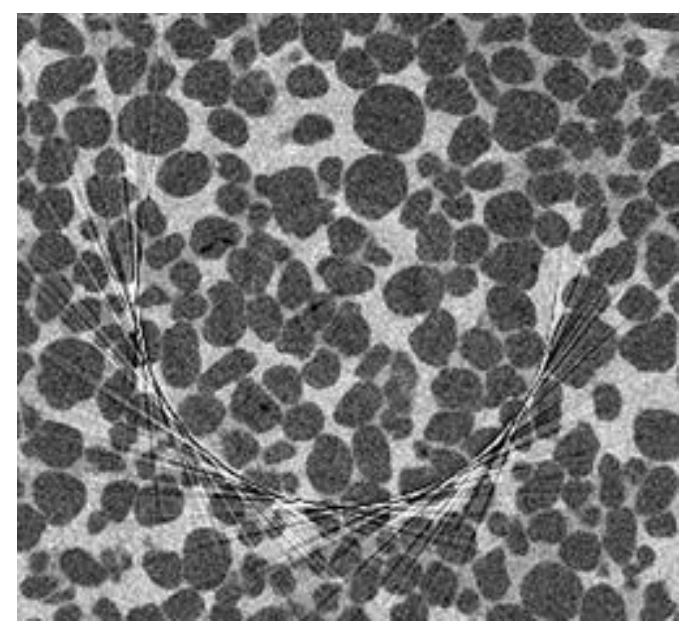

Figure 3. An example of ring-shaped artefacts in a microCT image.

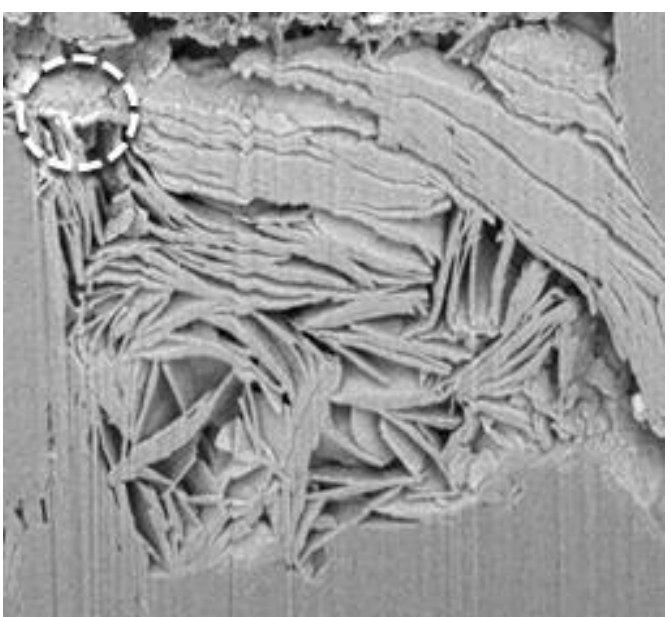

Figure 4. An example of bright pore edges and curtaining in a FIB-SEM image.

Typical X-ray microtomography images are $2000^{3}$ voxels or larger. FIB-SEM images can have similar size. However, the sample or region of interest can occupy only a part of such image. Further mathematical simulations usually require a cubic fragment with a side from 400 to 1000 voxels. Therefore, it is necessary to extract a small fragment of the best quality (in particular, with the least number of artefacts) from the original 3D image, which will be used for subsequent analysis and calculations. Currently, the operator usually subjectively evaluates the quality and does not have visualization tools to select the most suitable area. Thus, there is the problem to evaluate the quality of local fragments of three-dimensional tomographic images and visualize such estimations.

In the field of image processing and analysis, several non-reference (blind) quality criteria have been proposed at the moment. Such measures make it possible to evaluate quality based on only the image itself without marked reference data. They are usually developed for twodimensional photographs of nature, architecture, and everyday scenes, their applicability for assessing the quality of microCT or FIB-SEM images is unevaluated. Nevertheless, we assume that it makes sense to analyse the quality of the slices of microCT and FIB-SEM images using existing non-reference criteria to select $3 \mathrm{D}$ fragment of the highest quality.

Sometimes, the local quality of two-dimensional images is characterized with heat maps, where the brightness or colour level of each pixel in the image indicates the quality of the local fragment. For example, in [5] a heat map visualizes the quality of fingerprint images, and in [6] it is used for images of sample slides. However, this method is ineffective for threedimensional images, as it requires significant time to look through the inner parts of the image. In addition, we need to evaluate the quality of the local fragments with various sizes, because the quality, among other factors, can influence the choice of the fragment size for further modelling. In general, we can state that at the moment the problem of visualization of local quality of $3 \mathrm{D}$ images has not been solved.

In this paper, we propose an approach to the quantitative evaluation of the quality of slices and fragments of a three-dimensional tomographic image and, also, a method for its visualization to facilitate selection of a fragment for subsequent quantitative analysis of the sample structure and mathematical simulations.

\section{Existing non-reference metrics of image quality}

The paper [7] gives the taxonomy of existing non-reference methods for assessing the quality of photographs. They can be divided into two main categories: specific, which are intended for a particular defect, and general. Specific quality metrics are used, for example, to estimate noise level [8], sharpness [9], blur [10], as well as artefacts of lossy compression. Such quality assessments often precede the correction of the corresponding defect: sharpness is evaluated 
to find parameters for blur correction, and the level of JPEG artefacts is assessed to improve the quality of compressed images [11]. However, most specific metrics are designed for ideal distortions, for example, only Additive White Gaussian Noise (AWGN) is analysed. Also, these quality criteria imply that the corresponding defects prevail over others, while real images contain several types of distortion at the same time. Therefore, the use of such particular metrics should come only after a thorough analysis of possible defects for a given type of image and the mutual influence of these distortions.

Universal quality metrics are usually based on machine learning: the first step is extracting numerical features from the images, and then the regression model is trained to match the features with the assessments of observers $[12,13]$. The training was held on photographs from the LIVE data set [14], which contains 29 undistorted images and their 779 copies affected by one of five distortions: additive white Gaussian noise, Gaussian blur, JPEG compression artefacts, JPEG2000 compression artefacts, brightness and contrast changes. In some cases, models were adjusted with additional sets of images with similar characteristics, for example, TID2008 [15].

We considered the following algorithms as candidates for assessing the quality of microCT image slices: BIQI (Blind Image Quality Index) [12], BRISQUE (Blind Referenceless Image Spatial Quality Evaluator) [13], OG-IQA (Oriented Gradients Image Quality Assessment) [16], NIQE (Natural Image Quality Evaluator) [17] and IL-NIQE (Integrated Local Natural Image Quality Evaluator) [18]. The BIQI algorithm [12] implements a two-stage approach for assessing image quality, which is based on the natural scene statistic (NSS) in the wavelet domain [19]. It is assumed that natural images have certain statistical properties, and various distortions change these statistical characteristics in such a way that the type and degree of the distortion can be predicted. At the first stage BIQI finds the most probable type of distortion and then quantifies the degree. Models for classification at the first stage and regression at the second are trained using the support vector machine (SVM) on data from the LIVE set. The BRISQUE method [13] uses features from NSS calculated in spatial domain [20]. One regression model for all types of distortion is also trained by SVM on the LIVE image set. The OG-IQA algorithm [16] analyses the structure of the orientation of image gradients. Distortion of natural images are assumed to change the orientations of local gradients in a predictable way. The AdaBoost algorithm for decision trees was applied to train the regression model used for all types of distortions from the LIVE dataset. The disadvantages of the above models include a small number of photographs and a limited number of deformations in the training set, which leads to a low generalization capability of the algorithms.

The NIQE quality assessment criterion [17] does not use distorted images for training but constructs the multivariate Gaussian distribution (MVG) of NSS features calculated in the spatial domain [20]. The image quality is defined as the distance between its MVG and the reference MVG obtained from training on the attributes of undistorted images from the LIVE set. IL-NIQE [18] uses a similar idea but operates with RGB colour photo channels and with fragments that have significant intensity changes. We suppose that the IL-NIQE method can be effective for a comparative assessment of quality of microCT and FIB-SEM image slices. Firstly, it is sensitive to defects that are common to all types of images, such as noise and blur. On the other hand, a number of specific artefacts, for example, ring artefacts in microCT and curtaining in FIB-SEM images also affect this criterion, since they modify the MVG model of the undistorted image.

\section{Choosing the algorithm for assessment of quality of tomographic images}

We considered the tomographic images of sandstone to select the most suitable algorithm for the non-reference quality assessment. We chose 10 images of the same sample scanned by several microCT systems in different modes. Three experts independently performed pairwise comparison of their quality. Then the experts' assessments were transformed into a con- 
tinuous scale [21] and compared to the estimates of the above algorithms based on the correlation coefficient. Figure 5 shows the central fragments of two images from this set.
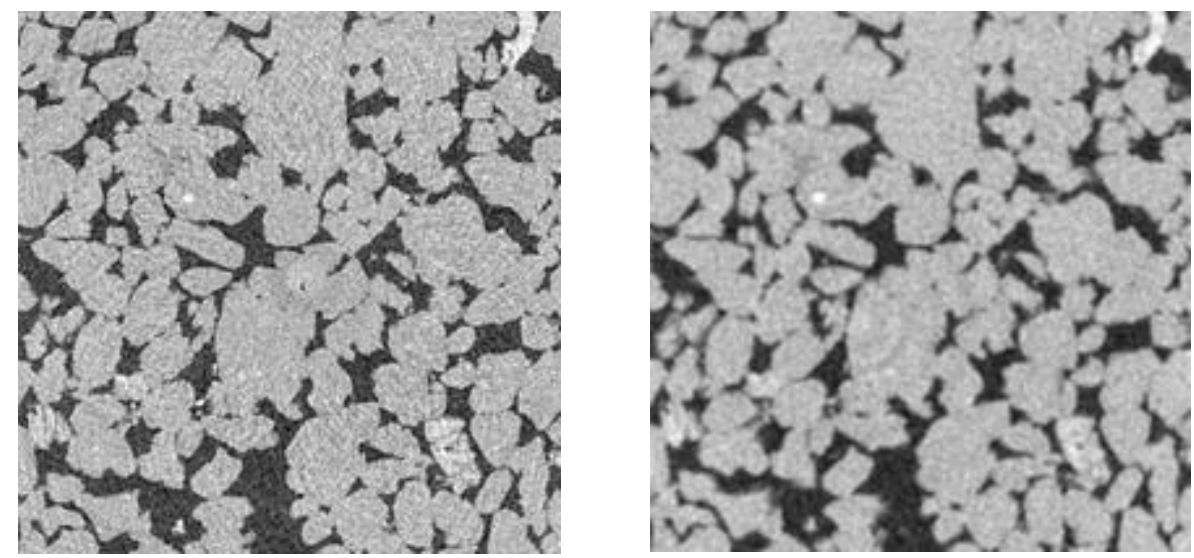

Figure 5. Examples of images with different quality from the test set.

Table 1 presents the values of the Pearson correlation coefficient between expert estimates and quality calculated with the considered algorithms for the test image set. We evaluated quality for the image of the entire slice and for its central part (25\% of the image were excluded from each side). This is because edges of the image often contain areas not related to the sample.

The IL-NIQE algorithm applied for the central part of the image showed the best result. Possible reason is that this measure less than others relates to a fixed set of data distortions used for training, and also uses a simpler model, less prone to overfitting.

Table 1. Pearson correlation coefficient between expert estimates and considered quality criteria of microCT images.

\begin{tabular}{|l|l|l|}
\hline Algorithm & $\begin{array}{l}\text { Correlation coeffi- } \\
\text { cient } \\
\text { (for the whole slice) }\end{array}$ & $\begin{array}{l}\text { Correlation coeffi- } \\
\text { cient } \\
\text { (for the central part) }\end{array}$ \\
\hline BIQI & 0.03 & 0.86 \\
\hline BRISQUE & 0.69 & 0.43 \\
\hline NIQE & 0.63 & 0.16 \\
\hline IL-NIQE & 0.53 & $\mathbf{0 . 9 4}$ \\
\hline OG-IQA & 0.48 & 0.39 \\
\hline
\end{tabular}

We put AWGN with various standard deviations on the FIB-SEM image (figure 4), and then calculated normalized quality measures (figure 6). The BIQI and OG-IQA criteria do not stay monotonic with increasing noise variance, therefore, cannot represent the quality of FIBSEM images. 


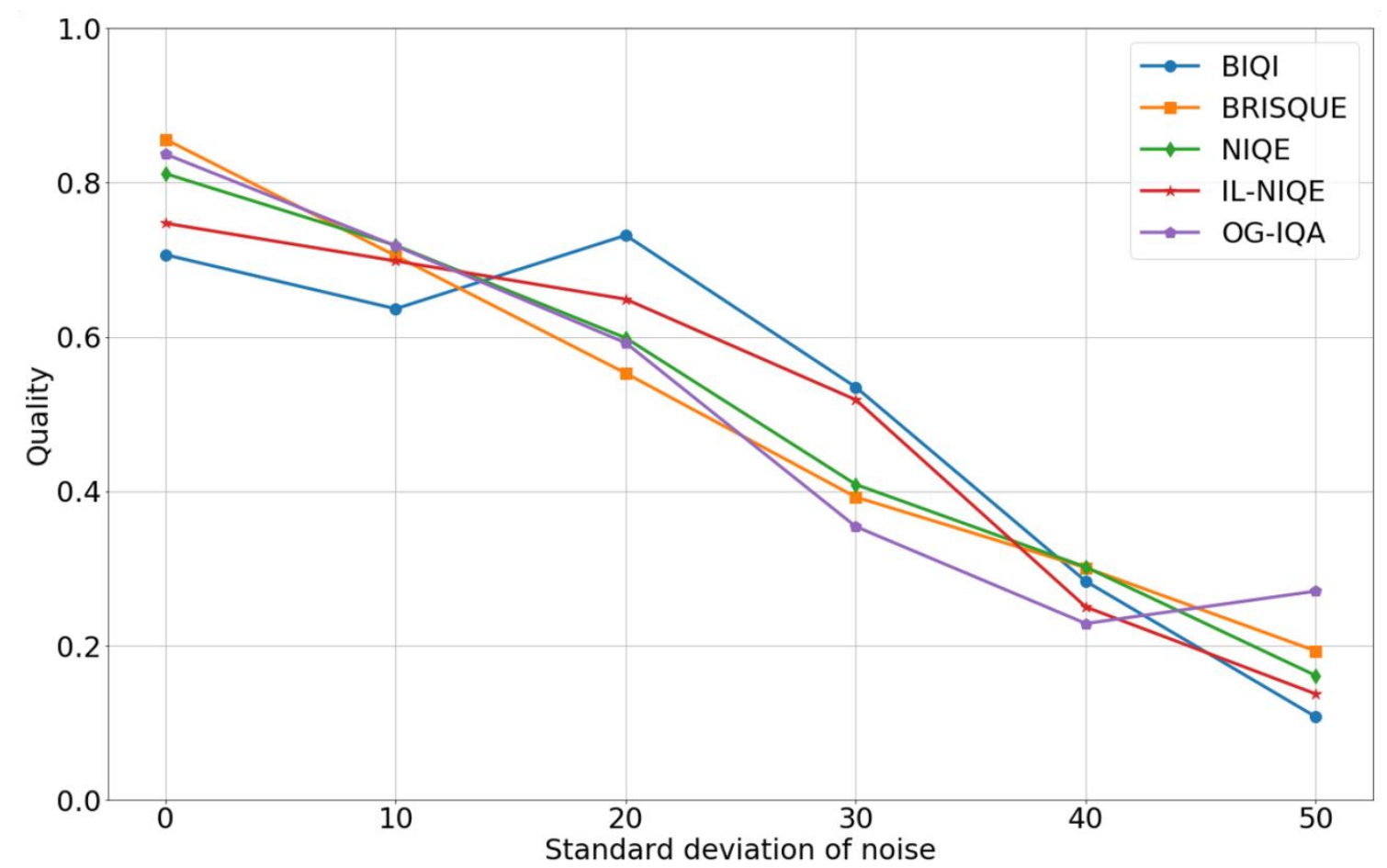

Figure 6. Quality value depending on standard deviation of additive white Gaussian noise applied to the FIB-SEM image.

Hereafter, we will use the IL-NIQE quality criterion to assess the quality of each slice, since it correlates better with the expert opinion on the quality of microCT images, and decreases monotonically with increasing noise in FIB-SEM images

\section{Visualization of quality of $3 \mathrm{D}$ tomographic images}

Three-dimensional microCT and FIB-SEM images are usually stored as slices in the (xy) plane and most often viewed in the same plane. Specialized software packages, such as Avi$\mathrm{zo}^{\circledR}$ (Thermo Fisher Scientific) [22], allow to visualize any projection and cross-section of $3 \mathrm{D}$ images in interactive mode. It is useful to analyse the central section of the image in the (xz) or (yz) plane in order to quickly evaluate the quality of tomographic images and select a fragment for further studies. In the case of X-ray tomography, such a central section allows to see which part of the image belongs to the sample. For FIB-SEM images, a side view immediately demonstrates how well the slices are aligned relative to each other. Relative displacements in the (xy) plane between adjacent slices lead to uneven pore edges on the side view (figure 7 ).

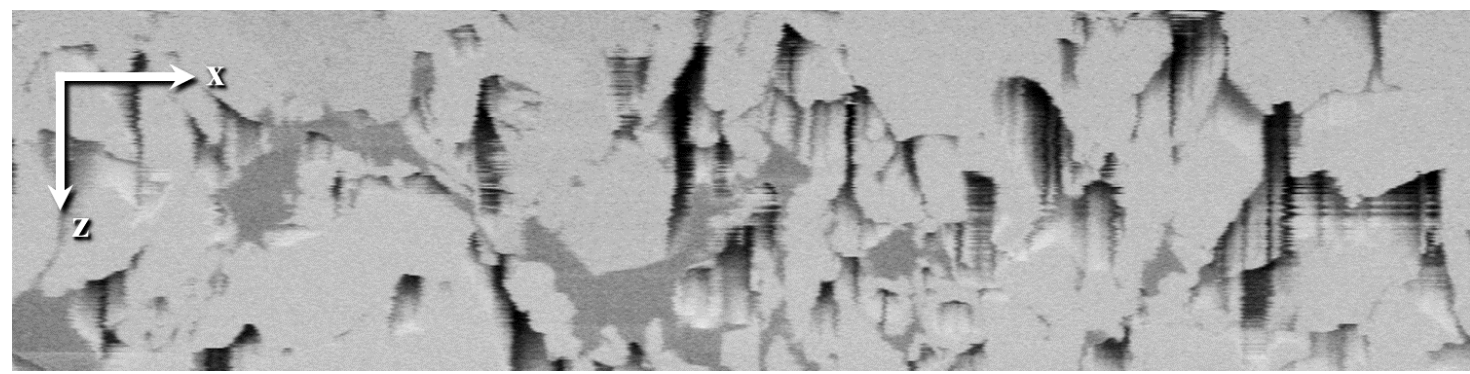

Figure 7. Cross-section of a FIB-SEM image in (xz) plane.

Another important parameter for choosing the best fragment is its homogeneity and representativeness (a measure of similarity between the fragment and the whole sample volume). To characterize the homogeneity of a three-dimensional image and the subsequent selection of a representative fragment, it is useful to introduce a measure of "similarity" of the slices. Let ${ }^{H_{c}}$ be the histogram of a set of several central slices. Let also ${ }^{H}$ be a histogram of an arbi- 
trary slice inside the region of interest. After normalization, both histograms ${ }^{H_{c}}{ }_{\mathrm{U}}{ }^{H}$ can be considered as probability distributions. For two discrete probability distributions, $H_{c}$ and ${ }^{H}$, similarity is defined as the unit minus Hellinger distance [23]:

$$
D_{\operatorname{sim}}=1-\frac{1}{\sqrt{2}} \sqrt{\sum_{i=0}^{255}\left(\sqrt{H_{c_{i}}}-\sqrt{H_{i}}\right)^{2}}
$$

It makes sense to show quality and similarity calculated for each slice over the image of the cross-section in the (xz) or (yz) plane (figure 8). This visualization method allows the operator to select the range of slices that is most suitable for further simulations within the digital rock workflow.

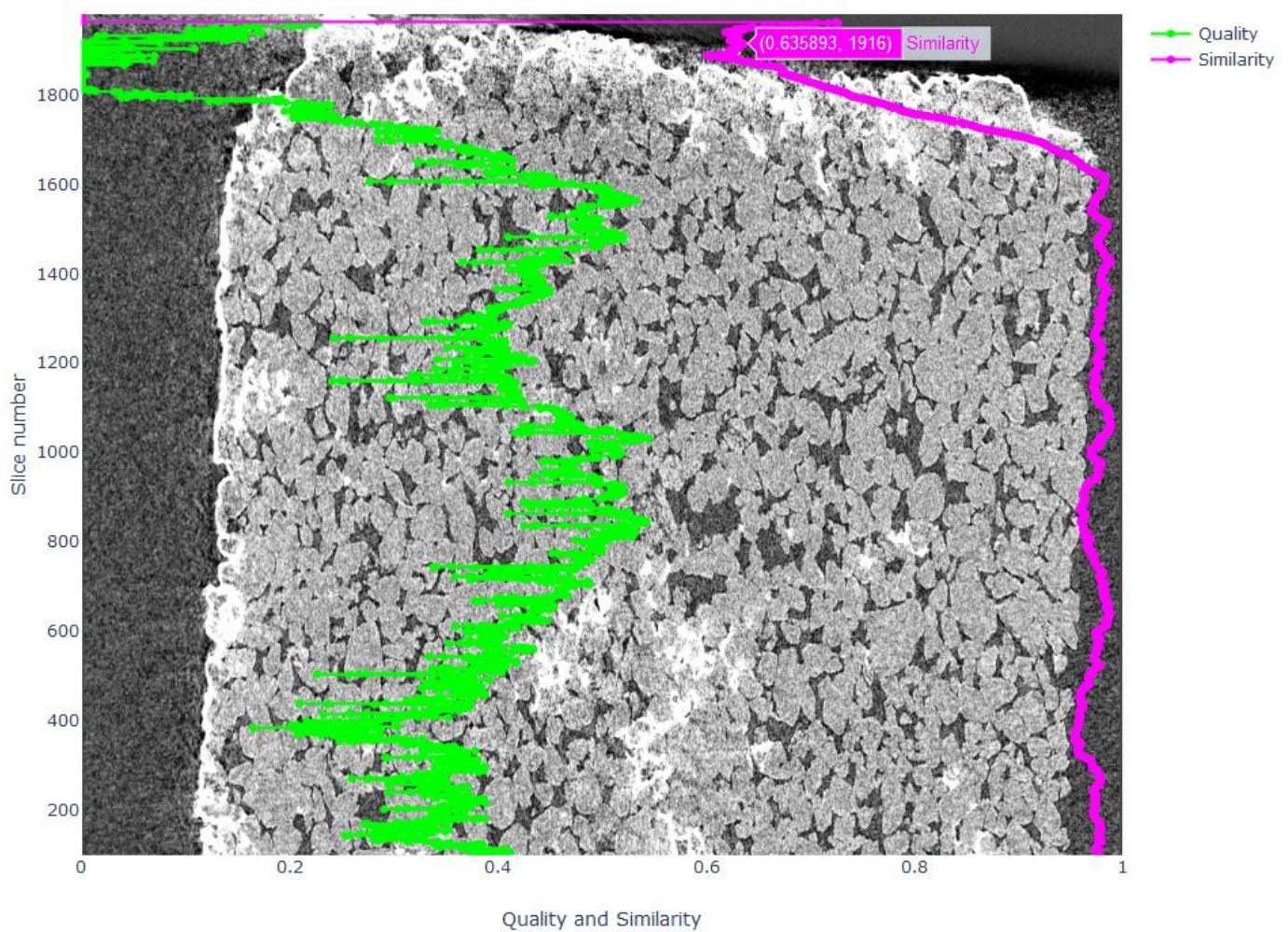

Figure 8. Quality measures and similarity depending on the slice number displayed over central section of the image in (xz) plane.

Another parameter while choosing the optimal fragment is its size. Depending on the objectives of the study and the structural features of the sample, fragments of various volumes are required (usually from $0.1 \mathrm{~mm}^{3}$ to $10 \mathrm{~mm}^{3}$ ). Thus, information on the integral quality of the selected image fragment depending on its size is of interest. For this purpose, we propose to evaluate and visualize the quality of a cubic image fragment with a centre in a given slice for fragments of various sizes. Quality for such a fragment is calculated as the average value from the quality measures of the slices crossing a given cube perpendicular to the $\mathrm{z}$ axis. Figurea shows the results. The z-coordinate of the centre of the inscribed cube is along the ordinate, and its size is along the abscissa. Colour represents the quality of a given cube. In fact, we plot a heat map along the $\mathrm{z}$-axis for each fragment size. The combination of such heat maps in a single drawing enables the operator to find the optimal position of the fragment in the original 3D image and at the same time to select its size. For the purpose of simplicity, the demonstrated approach implies that fragments are selected strictly from the centre of the slices. It is not difficult to find the optimal location of the cube in the (xy) plane. In this case, the heat 
map should display the maximum possible quality value for the given size and number of the central slice of the cube.

The Plotly library [24] allows to add an interactive view of the values on the graph (figure 7) and the combined heat map (figure 8).

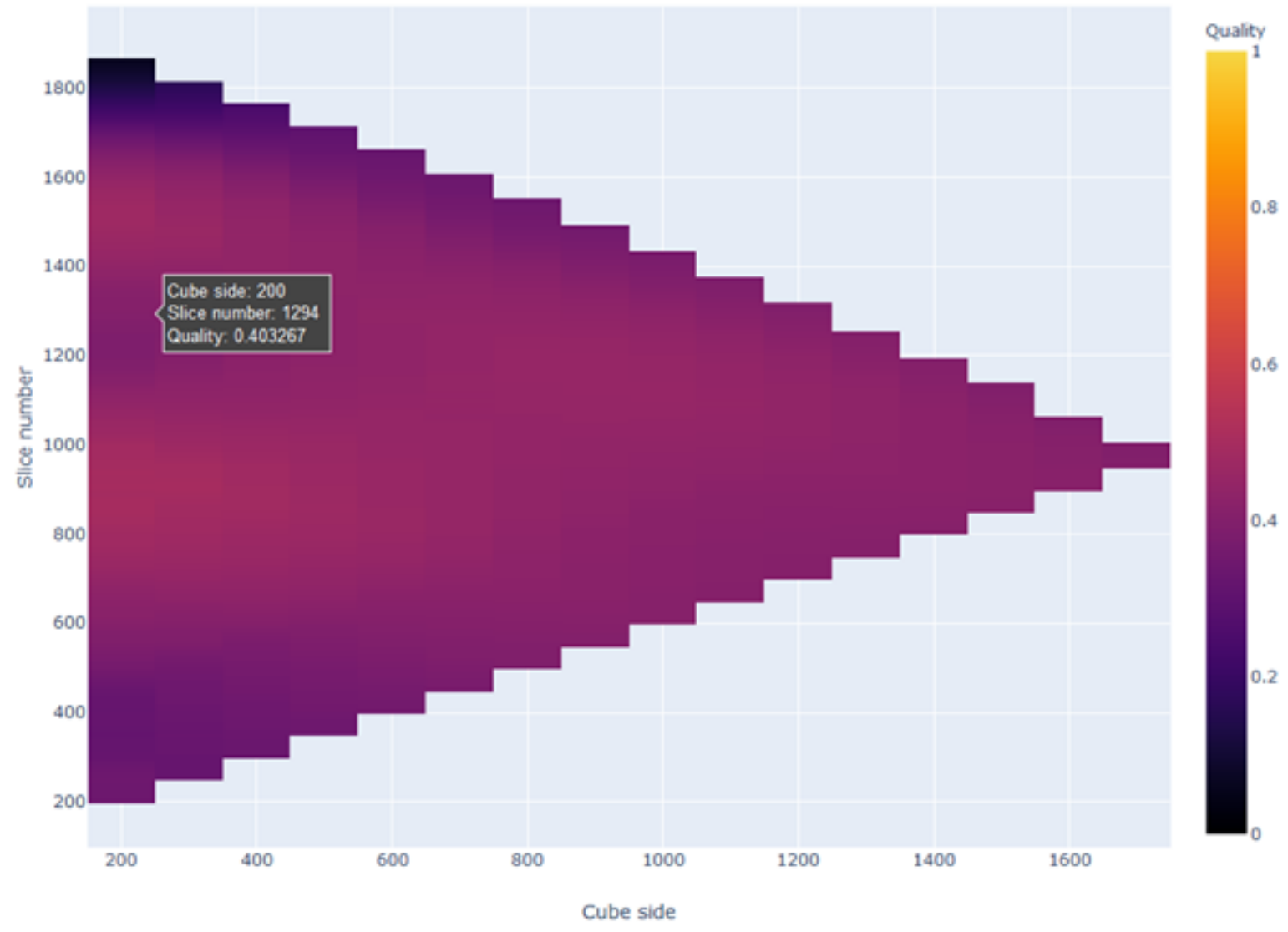

Figure 9. Combined heat map of quality of cubic fragment.

\section{Visualization for persons with colour blindness}

The disadvantage of colour graphs and heatmaps is that people with various forms of colour vision deficiency may have difficulty analysing colour information depending on the selected palette. When making colour graphs, one should avoid not only combinations of red and green, but also green and brown, yellow and light green, green and blue, blue and grey, blue and purple, etc. [25, 26] 10 shows a poor colour combination and what it looks like for people with deuteranopia - insensitivity to green. The image was obtained using the Color Blindness Simulator tool [27]. 

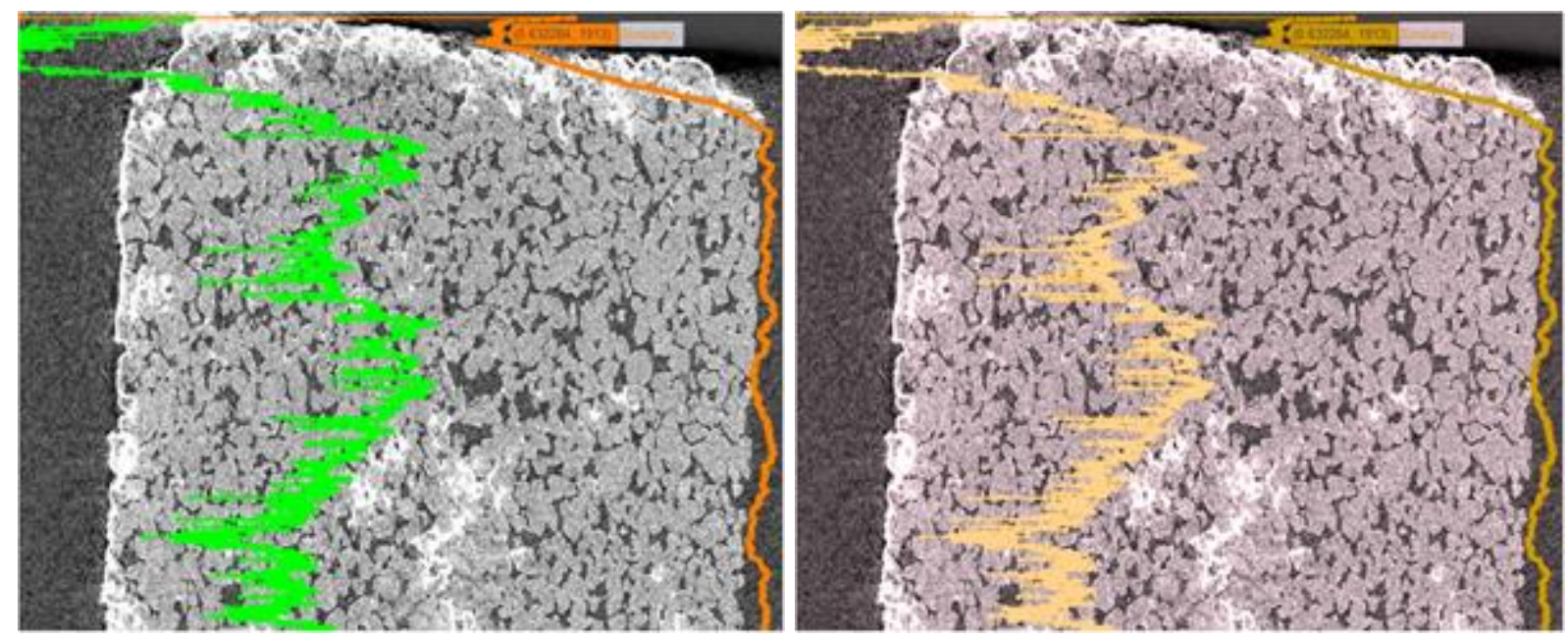

Figure 10. Appearance of colour graphs for people with normal vision (left) and with deuteranopia (right).

There are much fewer good combinations that would be suitable for most types of colour perception disorders at the same time. Such are, for example, green (o, 255, o) and magenta $(255,0,255)$, blue $(0,127,255)$ and orange $(255,127,0)$ (figure 11) [29]. In general, different markers, textures, colours of different intensities are preferable.

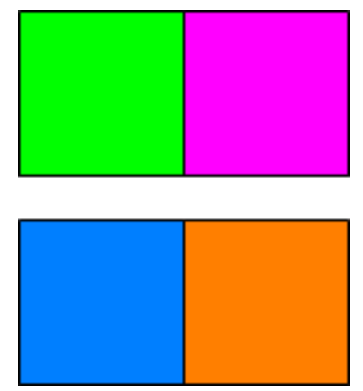

a)
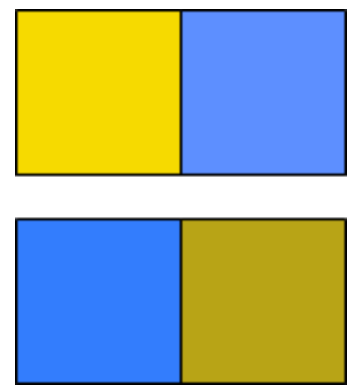

b)
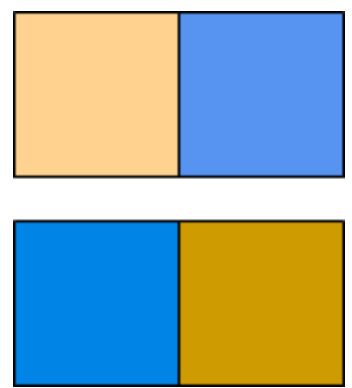

c)
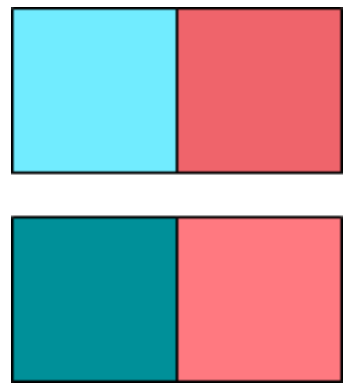

d)

Figure 11. Combinations of green and purple, blue and orange colours, as they are seen by people with: a) normal vision; b) protanopia; c) deuteranopia; d) tritanopia.

In case of heat maps, the most crucial distortion caused by colour vision deficiency is loss of monotony in the colour palette, when several completely different numerical values begin to correspond to one colour. However, even if a palette preserve monotony, in any case, it partially loses colour contrast over the entire interval or in some areas. If the data are located mainly in this distorted range, the heatmap may lose information. The best solution is to check the heatmaps using the colour blindness simulator and, if necessary, adjust the choice of the colour scale in accordance with the numerical range of data.

Regardless of colour blindness issue, palettes with linearly increasing intensity are recommended for building heat maps. Otherwise, the perception of false gradients is possible, since intensity is the most important characteristic for human vision. In addition, colour shades should be perceived equidistant in the colour space (i.e., to be "perceptually uniform") [29]. Popular palettes that satisfy these requirements are, for example, viridis from the matplotlib library [30], parula in the Matlab and a set of palettes cmocean designed for oceanographic applications [31].

With viridis palette, the heat map in figure 9 had a low colour contrast and became meaningless for some types of colour blindness. Therefore, we selected the inferno palette from the matplotlib library, which is also claimed to be "perceptually uniform". In general, it preserves content under various distortions, as well as in black and white, but it turns into shades of the 
same colour with one of the rarest colour vision disorders - tritanopia (0.0001\% of the population [32]) (Figure 12). A possible solution is to change the monitor settings, increase the contrast of the image, or automatically rescale the colour scale for each heat map individually.

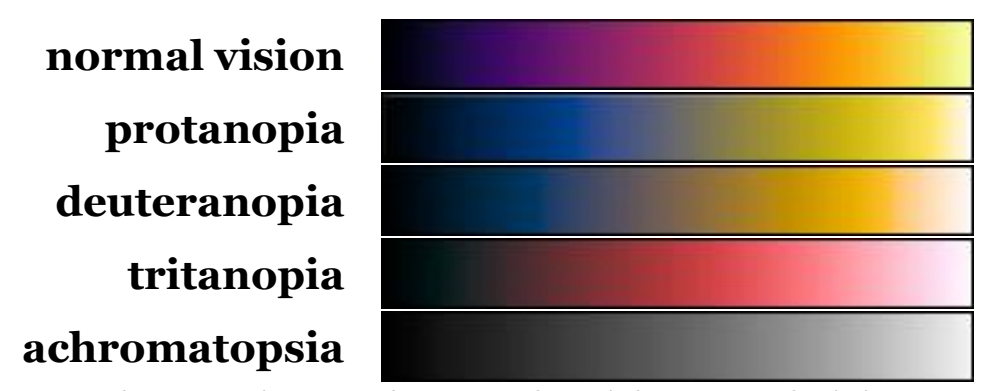

Figure 12. Inferno palette as it seen by people with normal vision and with various types of color vision deficiency.

\section{Conclusion}

We have investigated the applicability of the five existing non-reference quality measures for the evaluation of tomographic image slices. The IL-NIQE criterion coincided with experts' opinion the most. The probable reason is that this measure less than others relates to a fixed set of data distortions used for training, and uses a simpler model, less prone to overfitting. We have proposed the method to visualize quality of a three-dimensional tomographic image for the purpose of choosing the best fragment for subsequent mathematical modelling in the digital rock workflow. This approach includes demonstration of the cross-section of the image in the (xz) or (yz) plane and building on it the quality and similarity values for each slice, where the similarity is calculated using Hellinger distance. We have also proposed to build a combined heat map of the quality of cubic fragments with different sizes, inscribed in the original 3D image. Graphs and heat map are built considering their possible distortions in case of colour perception disorders. The main requirement when choosing a colour palette was the preservation of its monotony and information value for various types of colour vision deficiency. The matplotlib inferno palette is the most compliant with these requirements.

The disadvantage of the proposed approach is the inability to consider local artefacts in separate slices of the tomographic image when choosing the fragment of the best quality. This functional requires the development of an own algorithm for assessing the quality of analysed images, capable of detecting artefacts and evaluating quality in a local area. The development of an algorithm for the detection of specific artefacts of X-ray microtomography and FIBSEM images, as well as the visualization of a spatial map of artefacts in virtual reality, is the subject of our future research.

\section{References}

1. Koroteev D. et al. Direct hydrodynamic simulation of multiphase flow in porous rock. Petrophysics, 2014, vol. 55, № 04, pp. 294-303.

2. Berg C. F., Lopez O., Berland H. Industrial applications of digital rock technology. Journal of Petroleum Science and Engineering, 2017, vol. 157, pp. 131-147.

3. Buzug T. M. Computed Tomography: From Photon Statistics to Modern Cone-Beam CT. Springer-Verlag Berlin Heidelberg, 2008, 522 pp.

4. Holzer L., Cantoni M. Review of FIB Tomography. Nanofabrication Using Focused Ion and Electron Beams: Principles and Applications, edited by I. Utke, S. Moshkalev, P. Rusell, 2012, $752 \mathrm{pp}$.

5. Tabassi E., Wilson C. L. A novel approach to fingerprint image quality. IEEE International Conference on Image Processing 2005, 2005, vol. 2, p. II-37. 
6. SlideNet: Zhang T. et al. Slidenet: Fast and accurate slide quality assessment based on deep neural networks. 2018 24th International Conference on Pattern Recognition (ICPR), 2018, pp. 2314-2319.

7. Jenadeleh M., Masaeli M. M., Moghaddam M. E. Blind image quality assessment based on aesthetic and statistical quality-aware features. Journal of Electronic Imaging, 2017, vol. 26, № 4, pp. 043018.

8. Pyatykh S., Hesser J., Zheng L. Image noise level estimation by principal component analysis. IEEE transactions on image processing, 2013, vol. 22(2), pp. 687-699.

9. Safonov I. V. et al. Adaptive sharpening of photos. Color Imaging XIII: Processing, Hardcopy, and Applications, 2008, vol. 6807, p. 68070U.

10. Crete F. et al. The blur effect: perception and estimation with a new no-reference perceptual blur metric. Human vision and electronic imaging XII, 2007, vol. 6492, p. $64920 I$.

11. Safonov I. V., Kurilin I. V., Rychagov M.N., Tolstaya E.V. Adaptive Image Processing Algorithms for Printing. Springer Singapore, 2018. - 304 p.

12. Moorthy A. K., Bovik A. C. A two-step framework for constructing blind image quality indices. IEEE Signal processing letters, 2010, vol. 17(5), pp. 513-516.

13. Mittal A., Moorthy A. K., Bovik A. C. No-reference image quality assessment in the spatial domain. IEEE Transactions on Image Processing, 2012, vol. 21(12), pp. 4695-4708.

14. Sheikh H. R., Sabir M. F., Bovik A. C. A statistical evaluation of recent full reference image quality assessment algorithms. IEEE Transactions on image processing, 2006, vol. 15(11), pp. 3440-3451.

15. Ponomarenko N. et al. TID2008-a database for evaluation of full-reference visual quality assessment metrics. Advances of Modern Radioelectronics, 2009, vol. 10(4), pp. 30-45.

16. Liu L. et al. Blind image quality assessment by relative gradient statistics and adaboosting neural network. Signal Processing: Image Communication, 2016, vol. 40, pp. 1-15.

17. Mittal A., Soundararajan R., Bovik A. C. Making a "completely blind" image quality analyzer. IEEE Signal Processing Letters, 2013, vol. 20(3), pp. 209-212.

18. Zhang L., Zhang L., Bovik A. C. A feature-enriched completely blind image quality evaluator. IEEE Transactions on Image Processing, 2015, vol. 24(8), pp. 2579-2591.

19. Srivastava A. et al. On advances in statistical modeling of natural images. Journal of mathematical imaging and vision, 2003, vol. 18(1), pp. 17-33.

20. Ruderman D. L., Bialek W. Statistics of natural images: Scaling in the woods. Advances in neural information processing systems. - 1994, pp. 551-558.

21. Mantiuk R. K., Tomaszewska A., Mantiuk R. Comparison of four subjective methods for image quality assessment. Computer graphics forum, vol. 31(8), pp. 2478-2491.

22. Avizo web-site [Online]: https://www.thermofisher.com/ru/ru/home/industrial/ electron-microscopy/electron-microscopy-instruments-workflow-solutions/3d-visualizationanalysis-software.html (accessed on 01.03.2019).

23. Hellinger E. Neue begründung der theorie quadratischer formen von unendlichvielen veränderlichen. Journal für die reine und angewandte Mathematik, 1909, vol. 136, p. 210271.

24. Plotly web-site [Online]: https://plot.ly (accessed on 01.03.2019).

25. Rabkin E. B. Polychromaticheskie tablitsy dlya issledovania tsvetooshushenia [Polychromatic tables for research of colour perception]. Moscow, 1971, 174 p. [in Russian].

26. Okabe M., Ito K. Color Universal Design (CUD): How to make figures and presentations that are friendly to colorblind people [Online]: http://jfly.iam.u-tokyo.ac.jp/color (accessed on 01.03.2019).

27. Daniel Flück. Color Blindness Simulator [Online]: https://www.colorblindness.com/coblis-color-blindness-simulator (accessed on 01.03.2019).

28. Nuñez J. R., Anderton C. R., Renslow R. S. Optimizing colormaps with consideration for color vision deficiency to enable accurate interpretation of scientific data. PloS one, 2018, vol. 13(7), p. e0199239. 
29. Geissbuehler M., Lasser T. How to display data by color schemes compatible with redgreen color perception deficiencies. Optics express, 2013, vol. 21(8), pp. 9862-9874.

30. Matplotlib web-site [Online]: https://matplotlib.org/(accessed on 01.03.2019).

31. Thyng K. M. et al. True colors of oceanography: Guidelines for effective and accurate colormap selection. Oceanography, 2016, vol. 29(3), pp. 9-13.

32. Sharpe L. T., Stockman A., Jagle H. and Nathans J. Opsin Genes, Cone Pigments, Color Vision and Color Blindness. Color vision, edited by K. R. Gegenfurtner, L. T. Sharpe. Cambridge: Cambridge University Press, 1999. 